\section{AMYOTROPHIC LATERAL SCLEROSIS (ALS)}

CONCEPTS IN PATHOGENESIS ANI ETIOLOGY

Edited by

Arthur J. Hudson, MD hese essays examine some of the concepts on pathogenesis arising out of studies on the Western Pacific ALS. They also deal with new concepts on the 'classical' disease that is seen the world over.

Cloth 0-8020-3446-2 \$75.00

\section{UNIVERSITY OF TORONTO PRESS}

\section{ADVERTISERS INDEX}

Ciba/Geigy

Lioresal - obc, xxiii

Tegretol - iv, xi, xxi

Codman - Camino - vii

Dantec - v

Deprenyl - Eldepryl - xii, xiii

Dupont

Symmetrel - xvii, xxiii

Genderme

Axsaine - x, xxiv

Zostrix - xiv, xxiv

Janseen

Sibelium - viii, ix, xxii

Hoffmann - La Roche

Rivotril - xvi, xxiv

Nicolet Instruments - ii, xviii

Nihon Kohden - Xv

Parke Davis

Dilantin - ibc, $\mathrm{xx}$

Sandoz Canada

Parlodel - ifc, xix

Classified Ads - xxiii, xxiv, xxvi

\section{The Canadian \\ Journal of \\ Neurological \\ Sciences}

Rates:

Within Canada

Outside Canada

Please invoice

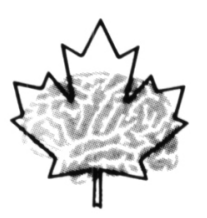

\section{Subscription Order Form}

\section{Le Journal Canadien des Sciences Neurologiques}

Name

Address

City Province

Country Postal Code

IMPORTANT: In Canada please return this form with a cheque or money order payable to the Canadian Journal of Neurological Sciences. All other areas, remit in Canadian funds drawn on a Canadian bank or US funds drawn on a US bank or by international money order.

Currency equivalents: $\quad$ Canadian $\$ 70.00=$ US $\$ 60.00$ Canadian $\$ 35.00=$ US $\$ 30.00$

Canadian $\$ 60.00=$ US $\$ 51.00$ Canadian $\$ 30.00=$ US $\$ 26.00$ 